TERRA. Revista de Desarrollo Local e-ISSN: 2386-9968

Número 6 (2020), 294-301

DOI 10.7203/terra.6.16221

IIDL - Instituto Interuniversitario de Desarrollo Local

\title{
Territorios y energías renovables. Proyecto de investigación "Sostenibilidad territorial del modelo energético bajo en carbono (TERRYER)"
}

\author{
Alba Ruíz Díez \\ Universidad de Sevilla (Sevilla, España) \\ albruidie@us.es \\ https://orcid.org/0000-0001-9374-6482 \\ Carolina del Valle \\ Universidad de Sevilla (Sevilla, España) \\ cdvalle@us.es \\ https://orcid.org/0000-0001-5327-0790 \\ María-José Prados \\ Universidad de Sevilla (Sevilla, España) \\ mjprados@us.es \\ https://orcid.org/0000-0002-3851-3405
}

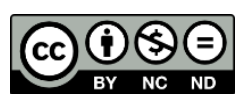

Esta obra se distribuye con la licencia Creative Commons Reconocimiento-NoComercial-SinObraDerivada 4.0 Internacional 


\section{SECCIÓN EXPERIENCIAS Y BUENAS PRÁCTICAS}

\section{Territorios y energías renovables. Proyecto de investigación "Sostenibilidad territorial del modelo energético bajo en carbono (TERRYER)"}

Resumen: La alarma climática que acecha actualmente al planeta, así como la preocupación por el agotamiento de los recursos fósiles, han irrumpido con fuerza en el panorama social, político y económico a escala mundial. Esto ha puesto en evidencia la necesidad de una transición hacia un modelo energético bajo en carbono, y para el que las energías renovables se han convertido en la alternativa más viable. Ante este escenario de transición energética, el proyecto de Investigación TERRYER se presenta como una herramienta de refuerzo del compromiso social para con la energía limpia, segura, sostenible y eficiente, a través de la participación activa de la población en la implantación de las energías renovables. A través de un equipo multidisciplinar, basado en los pilares de la comunicación, la transparencia y la inclusión, este proyecto persigue transformar el alcance del conocimiento científico sobre la implantación territorial sostenible de las energías renovables.

Palabras clave: Energía renovable, sostenibilidad, paisaje, territorio, transición energética.

Recibido: 9 de diciembre de 2019

Devuelto para revisión: 10 de diciembre de 2019

Aceptado: 23 de diciembre de 2019

Referencia / Citation:

Ruíz, A., del Valle, C., y Prados, M. J. (2020). Territorios y energías renovables. Proyecto de investigación "Sostenibilidad territorial del modelo energético bajo en carbono (TERRYER)". TERRA. Revista de Desarrollo Local, (6), 294-301. DOI 10.7203/terra.6.16221 


\section{INTRODUCCIÓN}

En las últimas dos décadas, los países de la Unión Europea vienen liderando un marcado proceso de reforma energética, introduciendo modificaciones en sus políticas en materia de energía para fomentar así la implantación de los recursos renovables como principal factor de suministro energético. Ahora bien, esta transición hacia un modelo energético bajo en carbono obliga a abordar un conjunto de retos que implican tanto a los ámbitos político y tecnológico como al científico.

Muchos han sido los estudios llevados a cabo desde las Ciencias Sociales, cuyas aportaciones han asentado las bases de la aproximación territorial a favor del nuevo modelo energético. La producción científica se ha centrado en cómo se lleva a cabo esta nueva transición en los paisajes de energía renovable (Frolova, Prados y Nadaï, 2015), junto con el estudio del comportamiento y la implicación de la población ante las energías renovables (European Comission, 2017), y el análisis de la política energética y la ordenación del territorio. Sin embargo, se ha puesto de manifiesto la falta de análisis relativos a los efectos o consecuencias concretos de la introducción de instalaciones renovables en territorios y poblaciones.

Ante este escenario, y desde la necesidad del estudio en mayor profundidad de las transformaciones y dinámicas socio-territoriales que conlleva el nuevo paradigma energético, surge el Proyecto de Investigación Sostenibilidad Territorial del Modelo Energético Bajo en Carbono (TERRYER).

Figura 1. Paisaje de energía renovable en Osuna, Sevilla: paisaje eólico

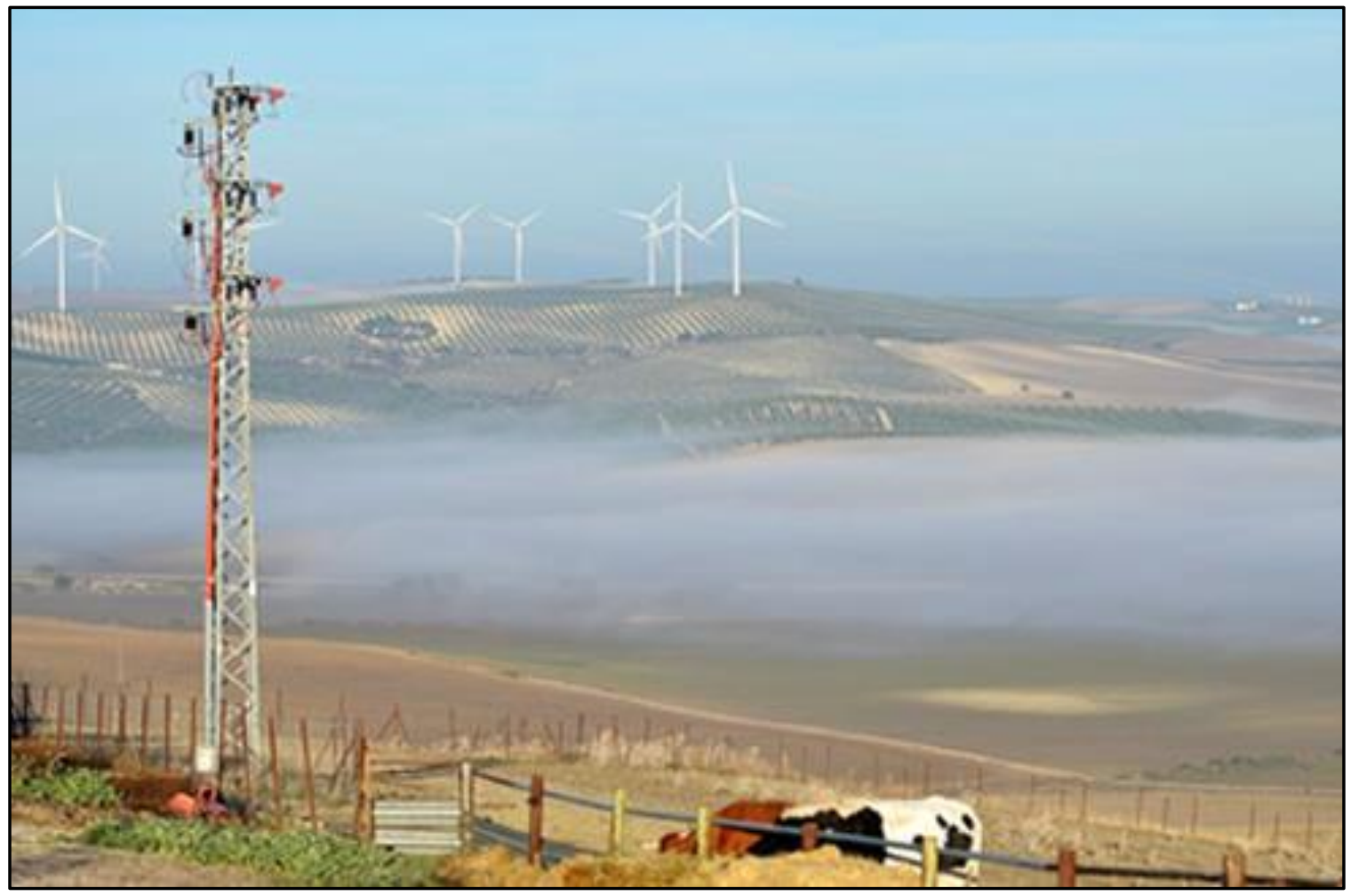

Fuente: Juan José González, Universidad de Sevilla. 2016 
Con este proyecto la comunidad científica aborda una nueva línea de investigación que amplía y transforma el alcance del conocimiento relativo a la implantación territorial y social sostenible de las energías renovables en la Península Ibérica. Dado que la mayor parte de los estudios europeos realizados se han centrado en países del Norte y Europa Central, TERRYER plantea la cuestión desde la óptica de dos países del Sur de Europa, España y Portugal, dada la importante cantidad, diversidad y capacidad de los recursos energéticos renovables que presentan estos países. Para ello, además del estudio del comportamiento e implicación de la población ante las energías renovables y de los procesos físico-ambientales asociados a su implantación en el territorio, TERRYER pretende realizar un análisis de la capacidad de dinamización económica de estas nuevas instalaciones (Figura 1).

\section{PROYECTO TERRYER: OBJETIVOS Y METODOLOGÍA}

Este proyecto se enmarca dentro del Programa Estatal de I+D+I Orientada a los Retos de la Sociedad, concretamente en el Reto número 3: "Energía Segura, Eficiente y Limpia". Este está coordinado por el Laboratorio Europeo de Ciencias Sociales de la Universidad de Sevilla, y cuenta con la participación conjunta de las universidades de Huelva, Murcia, Barcelona, Madrid y Lisboa, y las entidades empresariales española y portuguesa, Claner y Enercoutim, respectivamente. Se inició en enero del 2018 y su finalización está prevista para finales del año 2020. TERRYER plantea una investigación multidisciplinar, comparada e integradora a nivel territorial, que implica cinco casos de estudio en otros tantos ámbitos subregionales de los dos países indicados. Pretende romper con los planteamientos actuales relativos a los impactos socio-territoriales de las instalaciones de energía renovable y confirmar la capacidad que poseen las herramientas de análisis y reconocimiento territorial para el control de los impactos físico-ambientales asociados; la multifuncionalidad de las instalaciones renovables dada su aceptación en los dos países de estudio; y el papel de la innovación social, el consumo cooperativo y la gobernanza conjunta tanto en la implantación exitosa del nuevo modelo, como en la creación de relaciones positivas y beneficiosas entre promotores, población local y energías renovables.

Para la consecución de estas aspiraciones, el proyecto se articula en base a un conjunto de objetivos específicos que se resumen en:

- Explorar las razones de la amplia aceptación de las plantas de energía renovable y sus paisajes asociados en España y Portugal, identificando los puntos comunes en la implantación renovable exitosa, como claves ante el reto energético.

- Diseñar y aplicar herramientas metodológicas de evaluación y corrección de los impactos físico-ambientales de grandes instalaciones de energía renovable.

- Dimensionar los cambios positivos inducidos por las energías renovables a escala local a través de la generación de empleos, rentas complementarias y la diversificación económica.

- Propiciar la responsabilidad social y la gobernanza en el proceso de transición energética.

- Valorizar las energías renovables como base fundamental del desarrollo sostenible y la competitividad territorial. 
- Ampliar el conocimiento científico y la influencia en la formulación de políticas públicas.

Para el desarrollo de estos objetivos se aplican una serie de herramientas metodológicas (análisis bibliográfico y documental, trabajo de campo y laboratorio, entrevistas y encuestas, actividades de formación y transparencia y evaluación conjunta final), referidas a los casos de estudio concretos, los cuales incluyen tanto instalaciones renovables de diferentes tipologías energéticas -para el análisis de los procesos físicos y de negocio asociados- como cooperativas de energía -como referentes para propuestas de innovación social y gobernanza.

El plan de trabajo de TERRYER agrupa y organiza el desarrollo de las tareas en base a cinco bloques temáticos (Figura 2). El primero sienta las bases metodológicas, informativas, analíticas y conceptuales para el desarrollo y coordinación de todos los trabajos del proyecto. En el segundo bloque se analizan los emplazamientos de las instalaciones renovables para su posterior análisis físico-ambiental, cuyos resultados permitirán identificar disfuncionalidades y formular buenas prácticas para su mitigación, así como el estudio de la percepción social. El análisis de la capacidad dinamizadora y multifuncional se desarrolla en el bloque número tres. A continuación, el cuarto bloque se centra de lleno en el ámbito social, explorando métodos dirigidos a aumentar la innovación social y la gobernanza. Por último, el proyecto persigue desarrollar una red de actuaciones formativas y relacionales con empresas y comunidad universitaria que refuerce y ponga de manifiesto el protagonismo del análisis territorial de las energías renovables.

Figura 2. Plan de trabajo del proyecto TERRYER

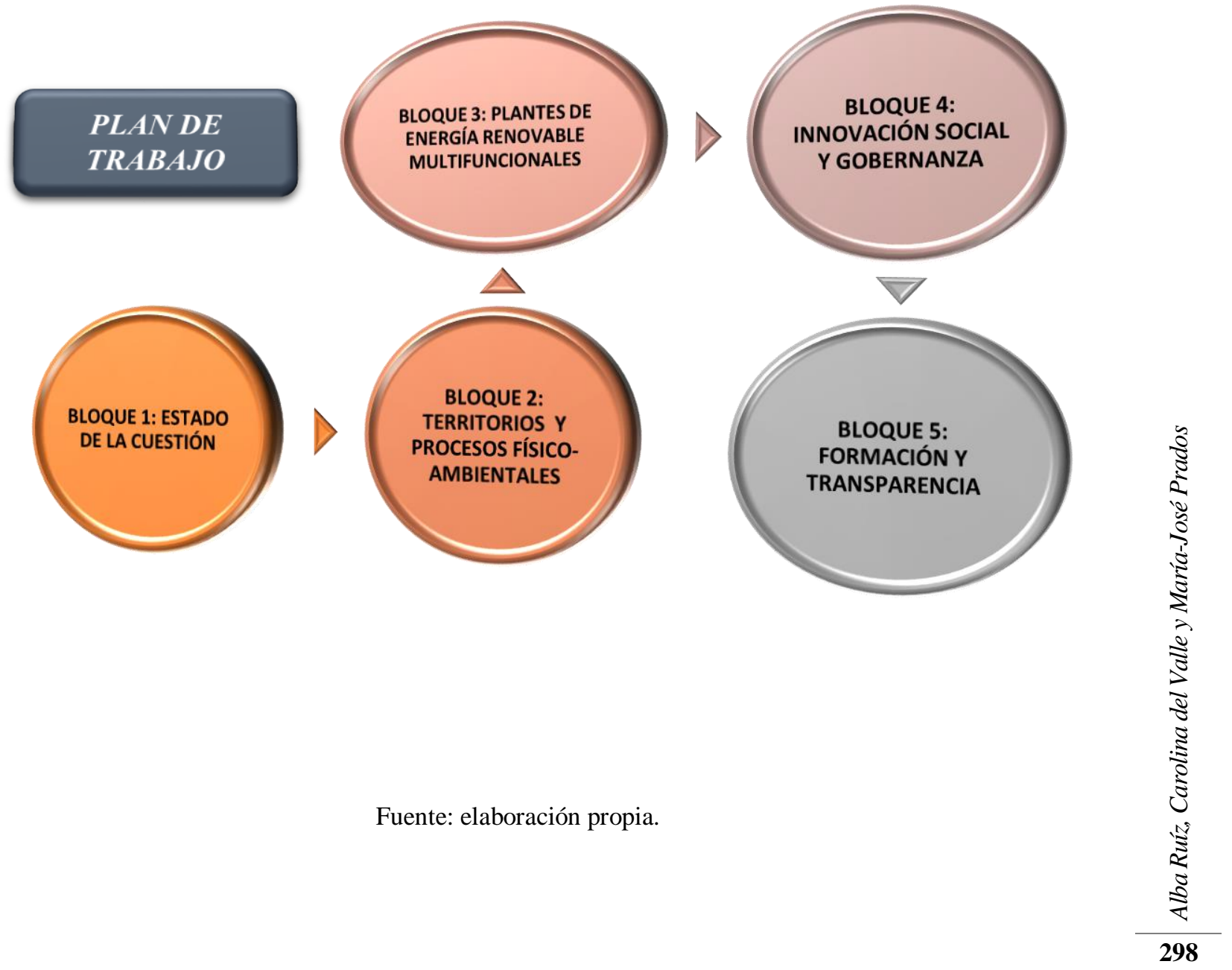




\section{PRIMEROS RESULTADOS}

Aunque el proyecto TERRYER se encuentra aún en fase de desarrollo, el carácter multidisciplinar del equipo que lo compone propiciará que sus resultados impliquen importantes impactos en diferentes escalas, desde el ámbito científico, hasta el social, pasando por el empresarial y político.

Las tareas realizadas hasta la fecha por el equipo de trabajo de TERRYER dentro de los diferentes bloques temáticos han posibilitado obtener una serie de resultados en relación a los objetivos de la investigación. Se han concretado un total de siete casos localizados en cuatro ámbitos de estudio de España y Portugal (Figura 3), y el análisis bibliográfico y documental ha permitido el establecimiento de tres marcos teóricos: Cambios físicoambientales, Innovación social y Modelos de negocio, sobre los que sustentar la investigación. Se ha llevado a cabo el análisis de los paisajes transformados por la presencia de las instalaciones renovables objeto de estudio, y de los procesos de cambios de usos de suelo. Han sido diseñadas las encuestas relativas al modelo de negocio y se ha iniciado el proceso de entrevistas con los actores implicados en la central hidroeléctrica de Capdella, Cataluña; en la central termosolar Gemasolar de Fuentes de Andalucía; en el parque eólico de Cantalejos, Osuna y en la planta fotovoltaica de Cantillana. Se han celebrado también, reuniones con distintas comunidades y cooperativas energéticas, como Coopernico y SOM Energía Sevilla. Este escenario está permitiendo obtener una evaluación ajustada de los beneficios y disfuncionalidades asociadas a los proyectos de energía renovable, así como valorar la implicación de la población local en el nuevo modelo energético.

En el ámbito de la difusión e internalización de los conocimientos generados, los resultados preliminares obtenidos en esta fase temprana de investigación han permitido que el equipo de proyecto participe en dos congresos con aportaciones multiautoría -International Conference on New Pathways for Comunity Energy and Storage, (Holanda, junio 2019) ${ }^{1}$ y XXVI Congreso de la Asociación de Geógrafos Españoles (Valencia, octubre 2019) ${ }^{2}$ - y el inicio de trabajos para la publicación de dos artículos. Otra principal aportación conseguida ha sido el diseño de una página web bilingüe ${ }^{3}$, como canal de comunicación y transparencia entre sociedad-investigaciónempresas, donde se comparte de forma atractiva y útil la información relativa al proyecto.

De este modo TERRYER está avanzando en el aumento del grado de concienciación de la población ante el cambio necesario de comportamiento respecto al consumo energético; la implicación de la ciudadanía a través de la generación e internalización de esquemas de participación pública; el fomento de nuevas metodologías que apoyen la investigación técnica y social relativa a las energías renovables; y la contribución a la formación de una nueva generación de investigadores en el ámbito de las energías renovables a diferentes escalas.

TERRYER prevé incluso impactos futuros indirectos, como podrían ser la mejora del funcionamiento del mercado energético, al generarse un mayor control del consumo por

\footnotetext{
${ }^{1}$ Delicado, A., Prados, M. J., Horta, A., Truninger, M. y Del Valle, C. (2019). Barriers and opportunities for community renewable energy in the south of Europe. International Conference on New Pathways for Community Energy and Storage. 6-7 June 2019, Groningen (The Netherlands).

${ }^{2}$ Del Valle, C., Delicado, A., Prados, M. J. y García, R. (2019). La participación pública en los procesos de implantación de las energías renovables en España y Portugal. XXVI Congreso de la Asociación Española de Geografía, 22-25 de octubre de 2019, Valencia.

${ }^{3}$ http://grupo.us.es/terryer/index.php
} 
parte de consumidores que se sumen de forma progresiva a las fuentes renovables, o la aparición de actividades vinculadas al turismo en el entorno de las instalaciones de energía renovable.

Figura 3. Localización espacial de los casos de estudio.

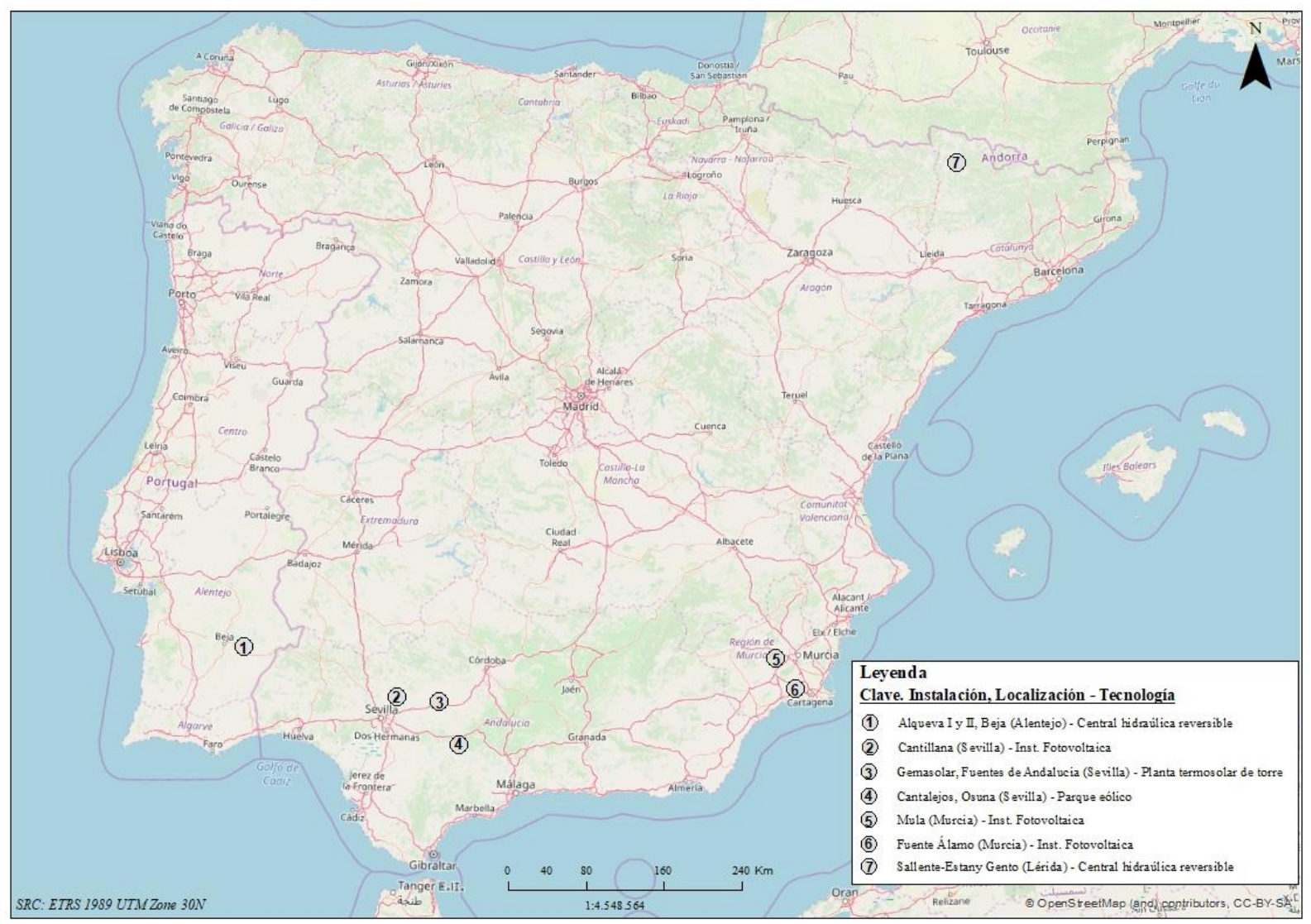

Fuente: elaboración propia a partir de datos facilitados por OpenStreetMap (OSM).

\section{REFLEXIONES PRELIMINARES}

Ante el complejo proceso de transición energética al que nos enfrentamos, es precisa la implicación activa y colaborativa de actores políticos, científicos, empresariales y sociales. Desde las Ciencias Sociales se generan los modelos analíticos que estudian el comportamiento de la población, y en este sentido el proyecto TERRYER aborda dichos patrones de comportamiento y percepción, abordando una nueva línea de investigación que propone nuevos métodos de innovación social y consumo energético de cara al futuro modelo bajo en carbono. Además de los ya comentados impactos y/o beneficios que se derivan de este proyecto, el estudio de la capacidad dinamizadora de los procesos energéticos renovables a nivel local, supondrá un punto de inflexión respecto a las potencialidades para estos ámbitos territoriales. La implantación de instalaciones renovables no es solo una manera de contribución positiva ante el escenario de transición actual, sino que también es una forma de reconocimiento, arraigo y desarrollo territorial.

Es necesario concienciar a la ciudadanía del proceso transitorio que ya ha dado comienzo, de la importancia y necesidad que conlleva, pero, sobre todo, hay que poner a su disposición las herramientas necesarias para abordarlo. 


\section{REFERENCIAS}

European Comission (2017). Scoping Paper for the Horizon 2020 Work Programme 2018-2020. Societal Challenge 3: Secure, clean and efficient energy. Recuperado de https://ec.europa.eu/research/participants/data/ref/h2020/wp/20182020/main/h2020-wp1820-energy_en.pdf

Frolova M., Prados M. J. y Nadaï, A. (ed.) (2015). Renewable Energies and European Landscapes. Lesson from Southern European Cases. Dordrecht: Springer. 